\title{
Cost-Effectiveness Analysis of Remdesivir Treatment in COVID-19 Patients Requiring Low-Flow Oxygen Therapy: Payer Perspective in Turkey
}

\author{
Ergun Oksuz (D) - Simten Malhan (D) - Mustafa Sait Gonen (D) • \\ Zekayi Kutlubay (D) · Yilmaz Keskindemirci (D) · James Jarrett • \\ Toros Sahin · Gokcem Ozcagli • Ahmet Bilgic • Merve Ozlem Bibilik • \\ Fehmi Tabak
}

Received: June 28, 2021 / Accepted: July 23, 2021 / Published online: August 11, 2021

(C) The Author(s) 2021

\section{ABSTRACT}

Introduction: This study aims to evaluate the cost-effectiveness of remdesivir compared to other existing therapies (SoC) in Turkey to treat COVID-19 patients hospitalized with $<94 \%$ saturation and low-flow oxygen therapy (LFOT) requirement.

Supplementary Information The online version contains supplementary material available at https:// doi.org/10.1007/s12325-021-01874-9.

E. Oksuz $(\bowtie)$

Department of Family Medicine, Faculty of

Medicine, Baskent University, Baglica Kampusu, Etimesgut, Ankara, Turkey

e-mail: eoksuz@baskent.edu.tr

S. Malhan

Department of Health Care Management, Faculty of Health Sciences, Baskent University, Ankara, Turkey e-mail: bmalhan@baskent.edu.tr

\section{S. Gonen}

Cerrahpasa Medical School, Istanbul University, Istanbul, Turkey

e-mail: sait.gonen@iuc.edu.tr

Z. Kutlubay

Cerrahpasa Medical School Hospital, Istanbul

University, Istanbul, Turkey

e-mail: zekayikutlubay@hotmail.com

Y. Keskindemirci

Istanbul University Hospitals, Istanbul, Turkey

e-mail: ykeskindemirci@gmail.com
Methods: We compared remdesivir as the treatment for COVID-19 with the treatments in the Turkish treatment guidelines. Analyses were performed using data from 78 hospitalized COVID-19 patients with $\mathrm{SpO}_{2}<94 \%$ who received LFOT in a tertiary healthcare facility. COVID-19 episode costs were calculated for 78 patients considering the cost of modeled remdesivir treatment in the same group from the payer's perspective. The incremental costeffectiveness ratio (ICER) per quality-adjusted life-year (QALY) was calculated for remdesivir

\section{J. Jarrett}

HEOR, Global Value and Access Europe, Gilead

Sciences, Foster City, CA, USA

e-mail: james.jarrett@gilead.com

T. Sahin · G. Ozcagli · A. Bilgic · M. O. Bibilik Gilead Sciences Turkey, Istanbul, TurkeyT. Sahin e-mail: Toros.Sahin@gilead.comG. Ozcagli e-mail: Gokcem.Ozcagli@gilead.com

A. Bilgic

e-mail:

ahmet.bilgic@gilead.comM. O. Bibilik

e-mail:

merveozlem.bibilik@gilead.com

F. Tabak

Department of Infectious Disease, Cerrahpasa

Medical School, Istanbul University, Istanbul, Turkey

e-mail: fehmitabak@hotmail.com 
versus the SoC for the population identified. For Turkey, a reimbursement threshold value between USD $8599(1 \times$ per capita gross domestic product-GDP) and USD 25.797 $(3 \times$ GDP $)$ per QALY was used.

Results: In the remdesivir arm, the length of hospital stay (LOS) was 3 days shorter than the SOC. The low ventilator requirement in the remdesivir arm was one factor that decreased the QALY disutility value. In patients who were transferred to intensive care unit (ICU) from the ward, the mean LOS was 17.3 days (SD 13.6), and the mean cost of stay was USD 155.3/day (SD 168.0), while in patients who were admitted to ICU at baseline, the mean LOS was 13.1 days (SD 13.7), and the mean cost of stay was USD 207.9/day (SD 133.6). The mean cost of episode per patient was USD 3461.1 (SD 2259.8) in the remdesivir arm and USD 3538.9 (SD 3296.0) in the SOC arm. Incremental QALYs were estimated at 0.174. Remdesivir treatment was determined to be cost saving vs. SoC.

Conclusions: Remdesivir, which results in shorter LOS and lower rates of intubation requirements in ICU patients than existing therapies, is associated with higher QALYs and lower costs, dominating SoC in patients with $\mathrm{SpO}_{2}<94 \%$ who require oxygen support.

Keywords: Remdesivir; COVID-19; Costeffectiveness; QALYs; Length of stay; Oxygen saturation

\section{Key Summary Points}

Why carry out this study?

The results of remdesivir trials found a significant reduction in time to recovery versus placebo for patients on supplemental oxygen arm as well as reduced progression to ICU and reduced requirement of invasive ventilation.

Remdesivir is currently not included in the COVID-19 treatment regimen in Turkey. Therefore, there is a need for a remdesivir pharmacoeconomic evaluation from the national repayer perspective.

\section{What was learned from the study?}

The current COVID-19 pandemic has once again brought up the importance of the urgent and efficient use of health resources.

Remdesivir does not result in significant additional costs in the treatment of COVID-19 in patient groups and subgroups with $\mathrm{SpO}_{2}<94 \%$ who are treated with low-flow oxygen therapy in the ICU.

Remdesivir is emerging as a cost-saving treatment option for a defined patient group.

\section{INTRODUCTION}

The World Health Organization (WHO) declared a Coronavirus Disease 2019 (COVID19) pandemic on March 11, 2020 [1]. Both developed and developing countries faced a lack of preparedness and intense pressure on resources as the pandemic unfolded, which has led to many unwanted outcomes. Given the pressure health systems are under because of COVID-19, it is important to ensure that healthcare resources are used efficiently. As new data and treatments become available, healthcare professionals worldwide are iteratively developing treatment algorithms to maximize patient benefit and minimize the impact on healthcare resources.

Increasing demand due to high rates of transmission of COVID-19 has nearly overwhelmed healthcare services. For example, in the first 12 days of the emergence of the disease in Italy, there was a $12 \%$ increase in intensive care unit (ICU) admissions [2, 3]. Hospitals had to rapidly increase their ICU bed capacity in the UK, with one hospital in London increasing capacity by $236 \%$ in the first wave [4]. At the beginning of the pandemic in Turkey, there were 40,000 ICU beds, and 18,000 had a mechanical ventilator. During the first peak of the pandemic in Turkey, ICU occupancy rates ranged from 44 to $70 \%$, and the ventilator 
occupancy rate ranged from 26 to $48 \%$ between cities. In 2020, Turkey began initiatives to increase ventilator capacity by nearly 35\%; existing hospital construction was sped up, and any newly built hospitals were designed to have high bed capacity that can easily convert into ICU beds [5].

The public health emergency highlighted the need to deploy each healthcare resource more efficiently. The increasing demand for healthcare services and its surge above the existing supply created a huge gap. The strains on the health system are, of course, not limited to capacity. For example, healthcare professionals are reporting burn-out. Other essential care has been indefinitely delayed, causing a lingering public health crisis and tension between the public and healthcare professionals [6].

Finding effective treatments for COVID-19 was an early priority in order to help with patient's recovery and alleviate symptoms as quickly as possible. Healthcare providers could work more effectively if patients are treated in a shorter time without any sequelae or a shorter length of hospital stay (LOS).

One of the first treatments authorized for emergency use through the respective processes of the United States Food and Drug Administration (FDA) (May 1, 2020, expanded August 28, 2020) [7] and European Medicines Agency (EMA) (July 3, 2020) [8] was remdesivir, an adenosine triphosphate analog, which is an effective antiviral against filoviruses, paramyxoviruses, pneumoviruses, and coronaviruses [9]. Remdesivir stops viral replication by inhibiting RNA-dependent RNA polymerase, a key viral enzyme [10]. The regulatory approval in the US and the EU was based on a randomized, doubleblind, placebo-controlled study conducted by the National Institute of Allergy and Infectious Diseases (ACTT-1) evaluating a total of 1062 patients hospitalized with mild, moderate, or severe COVID-19, who were randomized to receive either remdesivir $(n=541)$ or placebo $(n=521)$ in addition to standard of care (SoC) [11]. The results of this trial found a statistically significant reduction in time to recovery versus placebo for patients on supplemental oxygen arm (rate ratio for recovery 1.29; 95\% confidence interval [CI $1.12-1.49 ; p<0.001$ ) as well as reduced progression to ICU (RR 0.56; 95\% CI 0.49-0.61) and reduced requirement of invasive ventilation by $43 \%$.

Clinical studies and published meta-analyses [11-15] have shown that remdesivir treatment is effective. However, the treatment appears relatively expensive compared to other COVID19 treatments. Also, as remdesivir is currently not included in the COVID-19 treatment regimen in Turkey $[16,17]$ because of limited treatment experience with the product (there is currently a small ongoing clinical trial in Turkey), this study aims to evaluate the cost-effectiveness of remdesivir plus recommended care versus recommended care alone of treating COVID-19 patients with oxygen saturation $\left(\mathrm{SpO}_{2}\right)<94 \%$ who require low-flow oxygen therapy (LFOT) and ICU care from a national payer's perspective in Turkey. It also investigates how baseline characteristics may impact the cost of care.

\section{METHODS}

\section{Study Design}

This study utilized real-world data from a Turkish hospital and data from the literature to populate a cost-effectiveness model (CEM) (Fig. 1). The real-world data allowed for a detailed investigation into clinical factors associated with healthcare resource use (HCRU) and subsequent cost of caring for patients with COVID-19. These analyses were used to create baseline characteristics used in the economic model. The CEM was built using Microsoft Excel and used an average cost/average disutility approach for both groups to model four health states which corresponded to a certain LOS and hospital setting: General Ward-supplemental oxygen; ICU—supplemental oxygen; ICU-mechanical ventilation; death. A COVID-19 episode time horizon was assumed, and no discount rate was applied. The model assumes a cost-effectiveness threshold of $3 \times$ GDP [18]. The model considers COVID-19 patients hospitalized with $\mathrm{SpO}_{2}<94 \%$ and receiving LFOT in the ward and/or ICU of a tertiary healthcare 


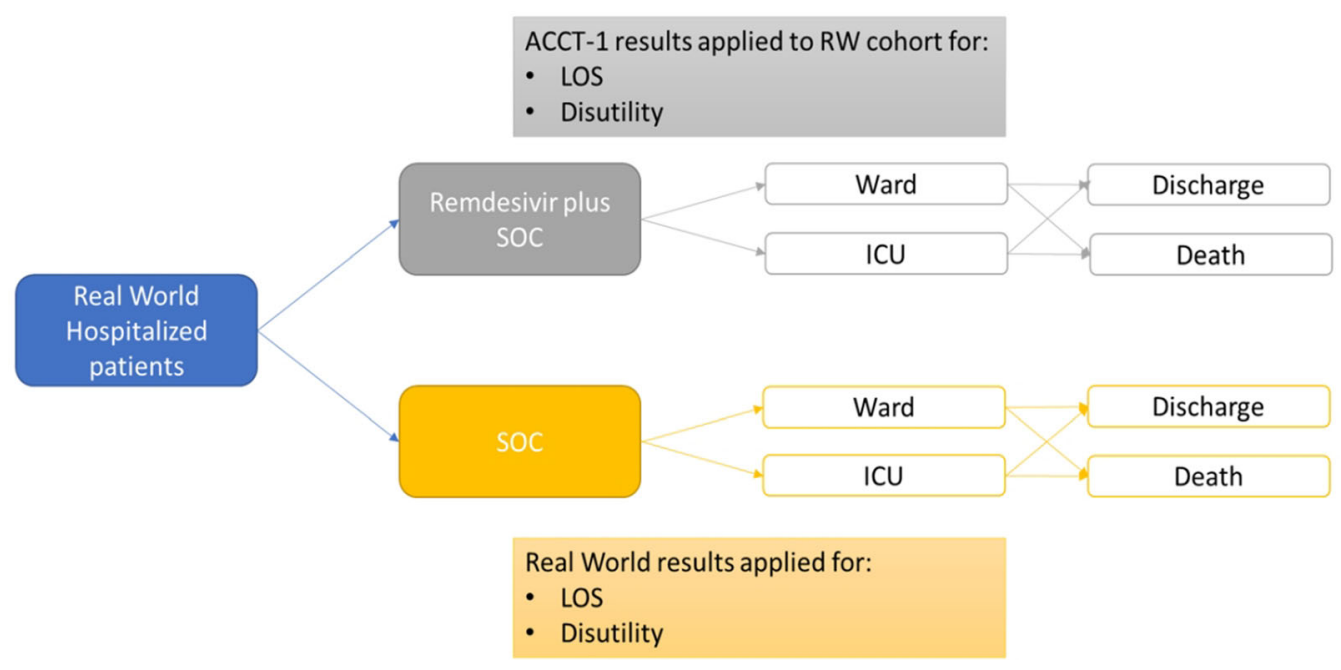

Fig. 1 Study design

facility in Turkey. The model considers a base case of treatment according to the Turkish guidelines. In Turkey, the treatment protocol for patients hospitalized with severe pneumonia who receive oxygen therapy is designed as hydroxychloroquine (HQ) $(2 \times 200 \mathrm{mg}$ tablet for 5-10 days) and/or favipiravir $(2 \times 1600 \mathrm{mg}$ loading dose followed by $2 * 600 \mathrm{mg}$ maintenance dose for 5-10 days) and/or $6 \mathrm{mg} /$ day dexamethasone (10 days) [16] compared to a scenario where patients also receive remdesivir as well as this treatment protocol.

The study was approved by the Ethics Committee of Istanbul University-Cerrahpasa (approval number: 830445809-604.01.04 A-08; approval date: 12.10.2020). Permission was obtained from the Republic of Turkey Ministry of Health and faculty administration for the use of patient data in our study (permission number: 2020-10-23T12-49-08; permission date: 23.10.2020).

All procedures performed in the study involving human participants were in accordance with the ethical standards of the institutional and national research committee and with the 1964 Helsinki Declaration and its later amendments or comparable ethical standards. Because of the retrospective nature of the study, the requirement for written informed consent was waived, and data used in this study were anonymized before their use.

\section{Data Sources}

To estimate the population parameters in the model, a real-world cohort of Turkish patients was obtained via the Hospital Data Management System (HDMS) of a tertiary hospital (Istanbul Cerrahpasa University Hospital) in Istanbul, Turkey. Patients were included if they had an $\mathrm{SpO}_{2}<94 \%$ who require LFOT and needed ICU care.

Health utility information was derived from the literature [19]. HRCU was derived from the HDMS data for patients. Healthcare costs were calculated from the national payer's perspective (Social Security Institution, SSI), and the analysis only focused on direct medical costs using the SSI reimbursement system to calculate COVID-19 ward and ICU costs. The cost of remdesivir is provided by Gilead.

\section{Outcomes}

Baseline demographics of the model population were derived from the HDMS real-world patient cohort. The statistical analysis of the real-world data allowed for an investigation into factors influencing the overall cost of care (see "Statistical Analysis" section below). The CEM presents results for the cost per patient, incremental cost, incremental quality-adjusted life-year (QALY), the incremental cost- 
effectiveness ratio (ICER), and incremental net benefit (INB). Data on the clinical effectiveness of remdesivir in terms of time to recovery and mortality were taken from the ACTT-1 trial [11].

In this study, the health state disutility weights were used, as calculated by the Institute for Clinical and Economic Review, which were also utilized in the study conducted by Kohli et al. [19] on the potential public health and economic value of a hypothetical COVID-19 vaccine in the USA. A QALY decrement, or a disutility weighted by the time spent with COVID-19 symptoms and being treated in hospital, was applied to all patients. The highest disutility weight of a patient who was hospitalized for 6 days because of COVID-19 was determined as 0.30 , the disutility weight of a patient hospitalized for 15 days in the ICU because of infection was 0.50 , and the disutility weight was determined as 0.60 if the patient needed mechanical ventilation in the ICU (Table 1). In this study, daily disutility values were calculated by adjusted LOS. In both arms, a disutility value of 0.04 /day was determined for patients who had a high level of ventilation requirement. A disutility value of 0.033 /inpatient days was used for hospitalized patients in the ICU who did not use a ventilator. Also, a disutility value of 0.05 /inpatient day was used for inpatients.

\section{HCRU and Costs}

Data on laboratory tests, $\mathrm{SpO}_{2}$, and imaging test results of the patients were analyzed from the HDMS. Patient characteristics were used to investigate the effects on costs or disutility values. Since the meta-analyses [20] reported that some inflammatory and hematologic markers are associated with the severity of COVID-19, we also took their impact on the cost into account in the analysis. For the purposes of the regression analysis (see "Statistical Analysis" section below), patients were categorized as the following: hematocrit levels $<35 \%$, lymphopenia $\left(<1000 / \mathrm{mm}^{3}\right)$, thrombocytopenia (platelet count $\left.<100 \times 10^{9} / \mathrm{l}\right), \quad$ neutrophilia (> 7700 neutrophils/ $\mu \mathrm{l}-11,000 \mathrm{WBC} / \mu \mathrm{l} \times 70 \%$ ), neutrophils/lymphocyte ratio (NLR) $\geq 2.95$ or $<2.95$, elevated fibrinogen $(>400 \mathrm{mg} / \mathrm{dl}$ ), elevated D-dimer ( $>4000 \mathrm{ng} / \mathrm{ml}$ ), elevated ferritin levels $(>500 \mathrm{ng} / \mathrm{ml})$, elevated serum creatinine concentration $(>1.5 \mathrm{mg} / \mathrm{dl})$, elevated urea $(>40 \mathrm{mg} / \mathrm{dl})$, elevated lactate dehydrogenase $(\mathrm{LDH})(>400 \mathrm{U} / \mathrm{l})$, elevated serum aminotransferases (alanine aminotransferase [ALT] and/or aspartate aminotransferase [AST] $>40$ units/l). At admission, patients were categorized based on C-reactive protein (CRP) levels of $<42 \mathrm{mg} / \mathrm{l}$ or $\geq 42 \mathrm{mg} / \mathrm{l}$. Patients were also categorized based on the presence of chest computerized tomography (CT) findings [16, 17, 20-23].

Within the SSI reimbursement system [24], the "payment based on procedure" method is used for certain healthcare services. In this payment scheme, healthcare costs are billed in packages. This system is similar to Healthcare Common Procedure Coding System (HCPCS) or Current Procedural Terminology (CPT Codes). In cases listed under this payment, the bed fee; examinations and consultations; operations and interventions; certain medications; consumables; laboratory, pathology, and radiology examinations; and anesthesia procedures are not invoiced separately. The SSI in Turkey has made payments to the hospitals based on daily procedures performed, under the name of "pandemic care payment," initially for patients

Table 1 Health state disutility parameters

\begin{tabular}{llll}
\hline Hospitalization & Disutility weights & Duration (days) & Daily disutility weights \\
\hline General ward with/without supplemental oxygen & 0.3 & 6 & 0.050 \\
ICU with/without supplemental oxygen & 0.5 & 15 & 0.033 \\
ICU + mechanical ventilation & 0.6 & 15 & 0.040 \\
\hline
\end{tabular}

$I C U$ intensive care unit 
who were real-time reverse transcription (PCR) positive and were hospitalized in the ICU (as of April 1, 2020). For COVID-19 ward hospitalization was USD 78.0/day, and for hospitalization in the ICU, based on the ICU levels, USD 26.0/day, USD 55.0/day, and USD 103.0/day. For patients who received a 'package payment,' it was impossible to separate the healthcare resource utilization. Therefore, these patients were assigned the package payment as a cost of treatment. They are referred to as procedure package payments in the analysis. Since remdesivir is not included in the national reimbursement list, the cost calculation was based on six vials/episode for all patients. Indirect costs were not considered for this analysis. All costs were converted to USD (USD $1.0=$ TRY 8.55).

\section{Statistical Analysis}

Descriptive statistics (mean and standard deviation [SD]) were used to determine outpatient examinations, emergency service (ER), general ward, and ICU admissions, laboratory and imaging tests, medical procedures, package procedures based on diagnosis, drugs, and medical supplies used. Total hospital costs were identified for treatment subgroups based on age ( $<65$ years and $\geq 65$ years); sex; symptoms at admission (fever, cough, and dyspnea); comorbidities; hematologic, coagulation, inflammatory, and biochemical biomarkers; and chest CT for pneumonia.

Continuous variables are expressed as mean with their SDs and median with their interquartile ranges (IQR); categorical variables are expressed as a number, percentage, and 95\% CI. We used $t$-test and summary $t$-test statistics to compare the characteristics of the groups and Levene and Hartley tests $\left(F_{\max }\right)$ for variance homogeneity.

Statistical calculations were made using SPSS 24.0 (IBM Corp, Armonk, NY) software. $p$ values $<0.05$ were considered statistically significant.

\section{RESULTS}

\section{Study Population}

Of the 233 patients identified in the HDMS during the pandemic's first peak in Turkey (March 11-July 31, 2020) a total of 78 patients met the selection criteria. The mean age of COVID-19 patients hospitalized in the ICU with $\mathrm{SpO}_{2}<94 \%$ receiving LFOT was 64.7 years (SD 16.9 ), and $48.7 \%$ of the patients were $\geq 65$ years of age; $67.9 \%$ of the patients were male; $70.5 \%$ had at least one comorbidity; $59.0 \% \quad(n=46)$ had positive chest CT at admission (Supplementary Table 1).

\section{Factors Influencing Cost of Care}

When the mean costs were compared based on patient characteristics, the cost per patient was USD 3889.2 for patients with elevated fibrinogen levels vs. USD 2451.1 for those with normal fibrinogen levels (mean difference USD 1438.1; 95\% CI 278.4-2597.8; $p=0.016$ ). The mean cost per patient was USD 4543.5 for patients who received steroids and USD 2762.5 for patients who did not receive steroids (mean difference USD 1781.0; 95\% CI 228.6-3333.4; $p=0.025)$. The cost was USD 5226.7 for patients who received mechanical ventilation vs. USD 2539.9 for those who did not receive mechanical ventilation (mean difference USD 2686.8; 95\% CI 982.0-4391.6; $p=0.003$ ). There were no significant cost differences among other groups (Supplementary Table 1).

\section{Cost-Effectiveness Analysis}

\section{Resource Use}

For patients receiving SoC, the mean LOS was 17.3 days (SD 13.6), and median LOS was 15.0 days (IQR 11.0); mean LOS in the ward was 5.9 days (SD 5.2), and median LOS in the ward was 4.0 days (IQR 5.0); mean LOS in the ICU was 13.0 days (SD 13.7), and median LOS in the ICU was 10.0 days (IQR 11.0). It was estimated that when patients were treated with remdesivir, the mean overall LOS was 10.2 days (SD 7.8), and the median was 9.0 days (IQR 7.0). The 
Table 2 Lengths of stay, services, need for ventilators, costs, and disutilities according to treatment arms

\begin{tabular}{|c|c|c|c|c|}
\hline & \multicolumn{2}{|c|}{ SoC-treated patients } & \multicolumn{2}{|c|}{ RDV + SoC-treated patients } \\
\hline & Mean (SD) & Median (IQR) & Mean (SD) & Median (IQR) \\
\hline \multicolumn{5}{|l|}{ Length of stay (days) } \\
\hline All patients $(n=78)$ & $17.3(13.6)$ & $15.0(11.0)$ & $10.2(7.8)$ & $9.0(7.0)$ \\
\hline Ward $(n=57)$ & $5.9(5.2)$ & $4.0(5.0)$ & $4.0(3.6)$ & $3.0(4.0)$ \\
\hline $\operatorname{ICU}(n=78)$ & $13.0(13.7)$ & $10.0(11.0)$ & $7.3(7.7)$ & $6.0(7.0)$ \\
\hline Ward and ICU $(n=57)$ & $18.4(14.7)$ & $15.0(10.5)$ & $11.0(8.4)$ & $9.0(5.5)$ \\
\hline Ward & $5.9(5.2)$ & $4.0(5.0)$ & $4.0(3.6)$ & $3.0(4.0)$ \\
\hline ICU & $12.5(15.0)$ & $9.0(10.5)$ & $7.0(8.5)$ & $5.0(5.5)$ \\
\hline \multicolumn{5}{|l|}{ ICU (directly) $(n=21)$} \\
\hline ICU & $14.4(9.3)$ & $10.0(13.0)$ & $8.1(5.2)$ & $6.0(7.0)$ \\
\hline Ventilator & & $n(\%)$ & & $n(\%)$ \\
\hline Ventilator $(-)$ & & $49(62.8)$ & & $60(76.9)$ \\
\hline Ventilator $(+)$ & & $29(37.2)$ & & $18(23.1)$ \\
\hline Costs (USD) & Mean (SD) & Median (IQR) & Mean (SD) & Median (IQR) \\
\hline \multicolumn{5}{|c|}{ Cost per diem per patient (USD) } \\
\hline Ward & $155.3(168.0)$ & $103.7(70.3)$ & $274.7(264.0)$ & $187.8(277.5)$ \\
\hline ICU & $207.9(133.6)$ & $179.0(89.3)$ & $450.4(198.5)$ & $415.6(139.3)$ \\
\hline \multicolumn{5}{|l|}{ Mean costs (USD) } \\
\hline Episode, per patient & $3538.9(3296.0)$ & $2537.0(3380.6)$ & $3461.1(2259.8)$ & $2675.3(669.8)$ \\
\hline \multicolumn{5}{|l|}{ Disutilities (QALYs) } \\
\hline Disutility, per patient & $0.515(0.417)$ & $0.410(0.340)$ & $0.341(0.282)$ & $0.251(0.240)$ \\
\hline
\end{tabular}

$\overline{I C U}$ intensive care unit, USD United States dollar, QALY quality-adjusted life-year, $S D$ standard deviation, IQR interquartile range, $S o C$ standard of care, $R D V$ remdesivir

mean LOS in the ward was estimated at 4.0 days (SD 3.6), and the median was 3.0 days (IQR 4.0); mean LOS in the ICU was 7.3 days (SD 7.7), and the median was 6.0 days (IQR 7.0). Completing remdesivir treatment with outpatient infusion in patients whose mean hospital stay is below the remdesivir treatment course is included in the cost. The number of patients switching from ward to ICU was $54.4 \%$ with SoC and $30.0 \%$ with remdesivir (95\% CI 26.6-33.0\%), which indicates a $24.4 \%$ reduction $(95 \%$ CI 21.3-27.7\%). Thirty-seven percent of patients $(n=29)$ in the recommended care arm required mechanical ventilation compared to $23.1 \%$ of the patients $(n=18)$ in the remdesivir arm (Table 2).

\section{Costs}

The mean cost per patient was USD 3538.9 (SD 3296.0), and the median was USD 2537.0. The mean cost was USD 559.1 (SD 674.1) for hospitalization in the ward, and the median cost was USD 388.6. The mean cost of ICU stay was 
Table 3 Distribution of ward and intensive care treatment costs and cost components of COVID-19 standard of care (USD)

\begin{tabular}{lccc}
\hline Cost components (USD) & Mean (SD) & Median (IQR) & Min-Max \\
\hline Physician costs & $3.9(4.7)$ & $2.7(3.9)$ & $0.0-26.2$ \\
Laboratory tests & $103.9(87.4)$ & $76.5(91.5)$ & $2.2-472.8$ \\
Imaging tests & $9.2(21.0)$ & $1.8(9.7)$ & $0.0-166.8$ \\
Beds & $69.5(66.2)$ & $51.7(65.7)$ & $5.7-378.1$ \\
Interventions & $56.5(61.2)$ & $39.9(53.1)$ & $0.0-369.9$ \\
Drugs & $449.0(494.1)$ & $253.5(597.8)$ & $11.9-2014.1$ \\
Medical supplies & $306.7(645.3)$ & $59.7(240.2)$ & $0.0-3583.2$ \\
Procedural packages & $2540.1(2571.6)$ & $1724.7(2505.9)$ & $0.0-15,632.9$ \\
Totals & & & \\
Ward & $559.1(674.1)$ & $388.6(840.4)$ & $0.0-4146.5$ \\
ICU & $2979.6(3426.7)$ & $1889.2(2842.2)$ & $15.1-19,642.4$ \\
Per patient & $3538.9(3296.0)$ & $2537.0(3380.6)$ & $170.0-19,750.3$ \\
\hline
\end{tabular}

$I C U$ intensive care unit, $U S D$ United States dollar, $S D$ standard deviation, $I Q R$ interquartile range, Min minimum, Max maximum

USD 2979.6 (SD 3426.7), and the median cost was 1889.2 (Table 3 ).

The mean daily cost per patient (per diem) in the ward was USD 155.3 (SD 168.0), and the median cost was USD 103.7 (IQR 70.3), while the mean cost in ICU was USD 207.9 (SD 133.6), and the median cost was USD 179.0 (IQR 89.3). The total costs included physician costs, laboratory tests, imaging tests, beds, interventions, drugs, medical supplies, and procedural packages. Procedural packages were the highest cost item within the total costs $(71.8 \%)$. It was followed by drugs (12.7\%), medical supplies $(8.7 \%)$, laboratory tests $(2.9 \%)$, beds $(1.9 \%)$, interventions $(1.6 \%)$, imaging tests $(0.3 \%)$, and physician costs $(0.1 \%)$ (Table 3$)$. Mean costs per patient for each item were: physician cost USD 3.9 (SD 4.7), laboratory tests USD 103.9 (SD 87.4), imaging tests USD 9.2 (SD 21.0), beds USD 69.5 (SD 66.2), interventions USD 56.5 (SD 61.2), drugs USD 449.0 (SD 494.1), medical supplies USD 306.7 (SD 645.3), and procedural packages USD 2540.1 (SD 2571.6) (Table 3).

The mean cost per patient with remdesivir was USD 3461.1 (SD 2259.8), and the median was USD 2675.3. Cost differences between the SoC and remdesivir arms were not significant in the subgroups (Supplementary Fig. 1, Supplementary Table 2 ). The highest positive difference was in patients with chronic renal disease (CRD) (USD 2632.6 with remdesivir vs. USD 2036.3 with SoC; summary $t=0.731 ; 95 \%$ $\mathrm{CI}-2346.1-1153.5)$ and in patients with thrombocytopenia (USD 2488.6 vs. USD 2081.8; summary $t=0.664 ; 95 \% \mathrm{CI}-1830.7-1017.1$ ). The highest negative difference was in patients who received steroid therapy (USD 4472.1 with remdesivir vs. USD 5226.7 with SoC; summary $t=-0.778 ; 95 \% \mathrm{CI}-1193.8-2703.0)$ and in patients treated with tocilizumab (USD 3475.2 with remdesivir vs. USD 3991.2 with SoC; summary $\quad t=-0.549 ; \quad 95 \%$ CI - 1381.9-2413.9) (Supplementary Fig. 1, Supplementary Table 2).

\section{Effectiveness}

For those patients treated with the SoC in a general ward, the disutility value was 0.520 compared to 0.286 for the remdesivir arm. For 
those patients treated in the ICU, the disutility was estimated at 0.502 for the SoC arm compared to 0.491 for the remdesivir arm. The percentage of patients who received mechanical ventilation was $37.2 \%$ in the SoC arm and $23.1 \%$ in the remdesivir arm, and the associated disutility values were 0.714 and 0.614 , respectively. The mean disutility value per patient was calculated at 0.515 for the SoC arm and 0.341 for the remdesivir arm. In this case, the incremental effectiveness was determined as 0.174 (SD 0.214) QALYs.

Differences in disutility were observed between the SoC arm and the remdesivir arm. Significant results were seen in the male gender subgroup (summary $t=2.584 ; p=0.011$ ) and in patients with pneumonia on chest CT at baseline (summary $t=2.626 ; p=0.011$ ). In addition, the disutility value differences between SoC and remdesivir arms were significant in patients with neutrophilia (summary $t=2.227$; $p=0.029$ ), lymphopenia (summary $t=2.014$; $p=0.047$ ), and NLR $\geq 2.95$ (summary $t=2.787$; $p=0.006$ ). The mean differences in disutility value were significant in groups of patients with $\mathrm{CRP} \geq 42 \mathrm{mg} / \mathrm{l}$ (summary $t=2.806 ; p=0.006$ ), elevated ferritin (summary $t=2.540 ; p=0.013$ ), and elevated fibrinogen (summary $t=2.520$; $p=0.013$ ). The disutility value differences were also significant in patients who presented with fever $(t=2.483 ; p=0.017)$ or dyspnea (summary $t=2.812 ; p=0.006)$. The mean differences in disutility value were statistically significant in patients who were treated with azithromycin (summary $t=2.055 ; p=0.047$ ), tocilizumab (summary $t=2.656 ; p=0.012$ ), HQ (summary $t=2.774 ; p=0.007$ ), lopinavir/ritonavir (summary $t=4.763 ; p=0.009$ ), anticoagulants (summary $t=2.887 ; p=0.005$ ), or favipiravir (summary $t=3.043 ; p=0.003$ ). The mean differences were also significant in patients with any comorbidities (summary $t=2.552 ; p=0.012)$, ischemic heart disease (IHD) (summary $t=2.603 ; p=0.019$ ), or hypertension (summary $t=2.077 ; p=0.043$ ) (Supplementary Fig. 2, Supplementary Table 3). Although the highest positive difference was observed in patients with asthma/chronic obstructive pulmonary disease (COPD) (0.321 with remdesivir vs. 0.617 with SoC; summary $t=0.846 ; 95 \%$ CI $-0.390-0.982$ ), it was not significant. The highest and significant differences were observed in patients treated with azithromycin (0.569 with SoC vs. 0.569 with remdesivir; summary $t=2.055 ; \quad 95 \%$ CI $0.012-0.510)$ and in patients treated with tocilizumab (0.574 with SOC vs. 0.326 with remdesivir; summary $t=2.656) ; 95 \% \quad \mathrm{CI}$ 0.065-0.431) (Supplementary Fig. 2, Supplementary Table 3$)$.

\section{Cost-Effectiveness Results}

In COVID-19 patients with $\mathrm{SpO}_{2}<94 \%$ and LFOT requirement, who were treated in the ICU in Turkey, the mean cost of an episode per patient was estimated at USD 3538.9 with SOC and USD 3461.1 with remdesivir. The incremental cost was USD - 77.8, and the incremental QALYs were 0.174. Compared to SoC, remdesivir treatment was observed as a costsaving (dominant) treatment option. When the results of INB analysis were evaluated, the INB value of remdesivir treatment against SoC was between 1576.6 USD and 4571.4 USD, and it was shown to be cost-effective (Table 4).

Table 4 ICER for remdesivir-ICU settings-COVID$19 \mathrm{SpO}_{2}<94 \%$ and $\mathrm{O}_{2}$ therapy

\begin{tabular}{lll}
\hline & Remdesivir & $\begin{array}{l}\text { Standard } \\
\text { therapy }\end{array}$ \\
\hline $\begin{array}{ll}\text { Cost per patient (mean, } \\
\text { USD) }(n=78)\end{array}$ & 3461.1 & 3538.9 \\
Incremental cost (USD) & -77.7 \\
Incremental effectiveness & 0.174 \\
$\quad$ QALYs) & \\
ICER (per QALY) & Dominant \\
INB & \\
QALY GDP $\times 1$ & 1575.6 \\
QALY GDP $\times 3$ & 4571.4 \\
\hline
\end{tabular}

ICER incremental cost-effectiveness ratio, ICU intensive care unit, $C O V I D-19$ coronavirus disease-19, $\mathrm{SpO}_{2}$ oxygen saturation, $\mathrm{O}_{2}$ oxygen, USD United States dollar, QALY quality-adjusted life-years, $I N B$ incremental net benefit, GDP per capita gross domestic product 
Patient outcomes matched with this patient modeled with the patient in the SoC arm were calculated head to head. Thus, the ICER plane was created by comparing the patients' costs and utility values in both treatment arms in the analysis. According to the cost-effectiveness analysis of remdesivir versus SoC, remdesivir is $61.5 \%$ cost-effective and provides $38.5 \%$ costsavings compared to SoC (Fig. 2).

According to the cost-effectiveness acceptability curve (CEAC), remdesivir starts to be cost-effective with a probability $>50 \%$ starting from USD 2500.0 willingness to pay (WTP). Also, the WTP threshold at the upper bound of USD $25,797.0$ appears to be a cost-effective treatment option with $90 \%$ probability (Fig. 3).

\section{DISCUSSION}

Remdesivir was compared with SoC in COVID19 patients in Turkey, who were admitted to the ICU with $\mathrm{SpO}_{2}<94 \%$ and received LFOT. In this model, the cost was calculated based on the actual hospital data, and the QALYs were generated based on the study conducted by Kohli et al. [19]; ICER was USD 1631.0/QALY. The threshold value was per capita income (GDP) $\times 3$ per capita for Turkey, i.e., WTP was USD $25,797.00$. Remdesivir was found to be a costeffective healthcare technology in this patient group compared to the SoC.

In a cost-effectiveness study conducted in South Africa, dexamethasone and remdesivir were compared to SoC in different scenarios for the treatment of COVID-19. Remdesivir was compared to SoC in patients who do not require ventilation, and dexamethasone was compared to SoC in patients who require ventilation; thus, 1111 deaths were found to be preventable compared to SoC. It was estimated that the prevention of these deaths would lead to a cost savings of USD 11.5 million [25].

In the study by Nichols et al., it was estimated that 3450 ICU beds were used for COVID-19 between June and December 2020. It has been stated that, without remdesivir, a total of 23,443-32,284 patients would use ICU beds and fully occupy the ICU capacity. With remdesivir, 36,383-47,820 patients at the first peak at the end of December were treated in ICU. The study reported that the use of remdesivir in COVID-19 patients could save many lives and may also provide potential ICU bed turnover by reducing the length of ICU stay [26].

Another study of 1643 patients receiving remdesivir reported that among the patients who were admitted in the last $24 \mathrm{~h}$ (LOS 3-14 days) and required mechanical ventilation, $36 \%$ had a LOS of $1-4$ days, $23 \%$ had a LOS of 5-8 days, and $41 \%$ had a LOS $\geq 9$ days. The authors reported that remdesivir resulted in a LOS reduction of 4 days in $41 \%$ of the patients; LOS was prolonged to complete 5-day treatment in $36 \%$, indicating that remdesivir use should be continued in the outpatient setting [27]. In a study conducted on 1062 hospitalized patients with low oxygen levels, the median time to recovery was 10 days in the remdesivir arm and 15 days in the placebo arm. The Kaplan-Meier estimates of mortality were $6.7 \%$ with remdesivir and $11.9 \%$ with placebo by day 15 and $11.4 \%$ with remdesivir and $15.2 \%$ with placebo by day 29 (hazard ratio, 0.73; 95\% CI 0.52-1.03). Serious adverse events were reported in $24.6 \%$ of the patients in the remdesivir group and $31.6 \%$ of the placebo group. Remdesivir was proven to significantly reduce the time to recovery in patients [11].

While evaluating the results of our study, it should be considered that it has some limitations. First, healthcare costs may differ between countries. Therefore, costs in other countries will differ from the values we calculate. In addition, the study was carried out from a single center. We used data from a tertiary healthcare institution. For this reason, it should be considered that there may be some differences between local and health service levels. At the time of the study, remdesivir was not an officially approved treatment for the treatment of COVID-19 in Turkey. Therefore, there are no country-specific remdesivir data. The study was conducted as a retrospective, and the non-randomized selection of patients is an important limitation. The study sample is also small because of the defined target patient population characteristics. We can say this is among our important limitations. Also, for COVID-19 


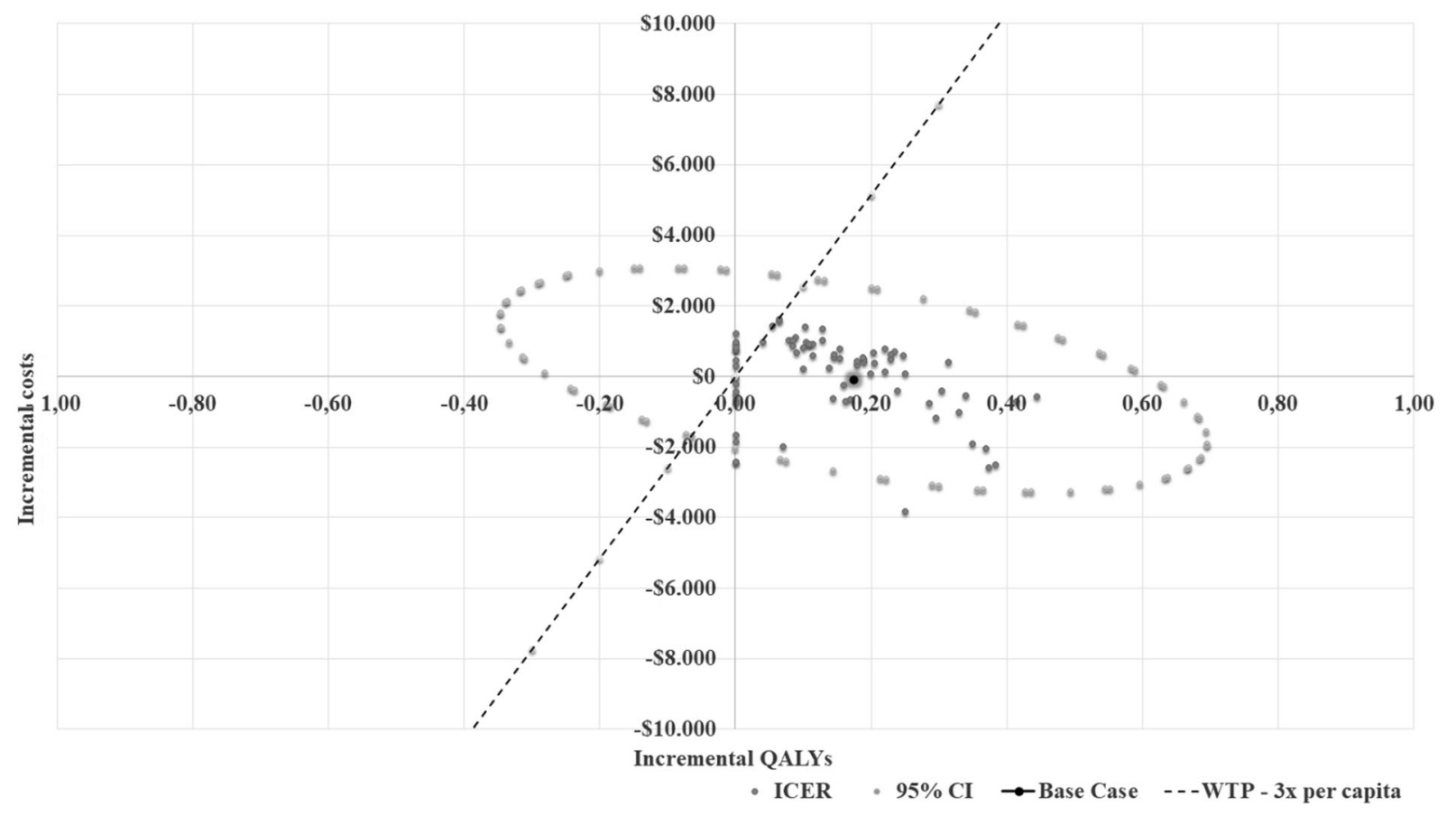

Fig. 2 ICER plane for remdesivir vs. standard therapy-ICU settings-COVID-19 $\mathrm{SpO}_{2}<94 \%$ and $\mathrm{O}_{2}$ therapy dataset

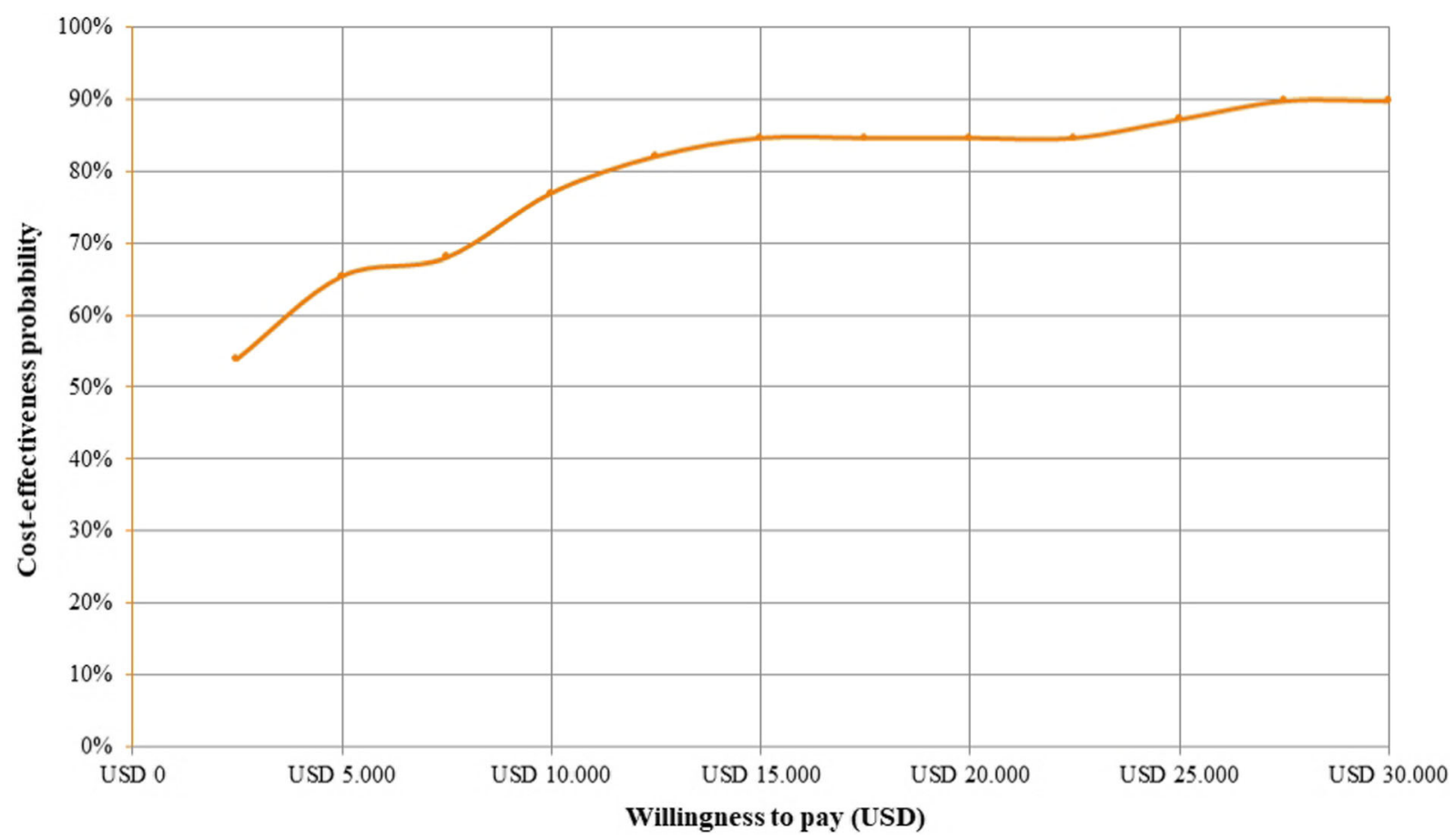

Fig. 3 Cost-effectiveness acceptability curve for remdesivir vs. standard therapy- ICU settings-COVID-19 $\mathrm{SpO}_{2}<94 \%$ and $\mathrm{O}_{2}$ therapy 
disease, there are no calculated QALY values for Turkey. Therefore, calculations were made using international disease-specific QALY values.

Turkey is faced with the risk of increased hospitalization rates due to the mutations in the virus and incomplete vaccination coverage. The increase in the number of patients, illness severity, hospitalization rates, and prolonged stay in ICU have negative impacts on healthcare systems during the pandemic. Remdesivir provides a faster bed turnover rate by shortening the LOS and reducing the ventilation requirement. This will allow more patients to access treatment in a shorter period. Remdesivir, which is reimbursed in many countries, stands out as an important COVID-19 treatment alternative for Turkey.

\section{CONCLUSION}

Remdesivir does not result in a significant additional cost in the treatment of COVID-19 in patient groups and subgroups with $\mathrm{SpO}_{2}<94 \%$ who are treated with LFOT in the ICU. Based on the cost associated with hospitalization, remdesivir has a lower cost in COVID-19 patients who have evidence of pneumonia on chest CT, in subgroups based on their hematologic (neutrophilia, lymphopenia, or elevated NLR) or inflammatory (elevated CRP, ferritin, or fibrinogen) biomarkers, in patients who presented with fever and dyspnea, as well as patients with comorbidities. Remdesivir, which results in shorter LOS and lower rates of intubation requirements in ICU patients, is associated with higher QALYs and lower costs, dominating SoC from the national payer's perspective in patients with $\mathrm{SpO}_{2}<94 \%$ who require oxygen support.

\section{ACKNOWLEDGEMENTS}

The authors thank the staff of the Cerrahpasa Faculty of Medicine \& the Republic of Turkey Ministry of Health for their devoted work throughout the pandemic. This study was sponsored by Gilead Sciences.
Funding. Gilead has provided financial support for the completion the full analysis and write up of the scientific data for publication. All authors had full access to the full data and the content of the publication reflects their views. Gilead has not been involved in any way in the drafting and/or review of the publication. The journal's Rapid Service Fee was funded by SIMER Arastirma Gelistirme Saglik Ekonomi ve Tek Egt Dan Ith Ihr San Tic AS.

Authorship. All named authors meet the International Committee of Medical Journal Editors (ICMJE) criteria for authorship for this article, take responsibility for the integrity of the work as a whole, and have given their approval for this version to be published.

Authors' Contributions. All authors were involved in the concept and design. Oksuz, Ergun: Data curation, Investigation, Writing, Visualization, Project administration. Malhan, Simten: Investigation, Writing, Visualization, Project administration. Gonen, Mustafa Sait: Resources, Software, Validation, Data curation, Methodology. Kutlubay, Zekayi: Resources, Software, Validation, Data curation, Methodology. Keskindemirci, Yilmaz: Resources, Software, Validation, Data curation, Methodology. Jarrett James: Writing-review \& editing, Visualization. Tabak, Fehmi: Investigation, Resources, Writing-review \& editing, Supervision, Project administration.

Disclosures. Ergun Oksuz, Simten Malhan, Mustafa Sait Gonen, Zekayi Kutlubay, Yilmaz Keskindemirci, and Fehmi Tabak have no conflicts of interest that are directly relevant to the content of this article. James Jarrett, Toros Sahin, Gokcem Ozcagli, Ahmet Bilgic, and Merve Ozlem Bibilik are employees of Gilead Sciences.

Compliance with Ethics Guidelines. The study was approved by the Ethics Committee of Istanbul University-Cerrahpasa (approval number: 830445809-604.01.04 A-08; approval date: 12.10.2020). Permission was obtained from the Republic of Turkey Ministry of Health and faculty administration for the use of patient data in 
our study (permission number: 2020-10-23T1249-08; permission date: 23.10 .2020 ). All procedures performed in the study involving human participants were in accordance with the ethical standards of the institutional and national research committee and with the 1964 Helsinki Declaration and its later amendments or comparable ethical standards. Because of the retrospective nature of the study, the requirement for written informed consent was waived and data used in this study were anonymized before its use.

Data Availability. The datasets generated and/or analyzed during the current study are not publicly available due to Turkish Personal Data Protection Law no. 6698 but are available from the corresponding author on reasonable request.

Open Access. This article is licensed under a Creative Commons Attribution-NonCommercial 4.0 International License, which permits any non-commercial use, sharing, adaptation, distribution and reproduction in any medium or format, as long as you give appropriate credit to the original author(s) and the source, provide a link to the Creative Commons licence, and indicate if changes were made. The images or other third party material in this article are included in the article's Creative Commons licence, unless indicated otherwise in a credit line to the material. If material is not included in the article's Creative Commons licence and your intended use is not permitted by statutory regulation or exceeds the permitted use, you will need to obtain permission directly from the copyright holder. To view a copy of this licence, visit http://creativecommons.org/licenses/by$\mathrm{nc} / 4.0 /$.

\section{REFERENCES}

1. Kinross P, Suetens C, Gomes Dias J, et al. Rapidly increasing cumulative incidence of coronavirus disease (COVID-19) in the European Union/European Economic Area and the United Kingdom, 1 January to 15 March 2020. Eurosurveillance. 2020;25(11):2000285.
2. Pecoraro F, Clemente F, Luzi D. The efficiency in the ordinary hospital bed management in Italy: an in-depth analysis of intensive care unit in the areas affected by COVID-19 before the outbreak. PLoS ONE. 2020;15(9):e0239249.

3. Rezoagli E, Magliocca A, Bellani G, Pesenti A, Grasselli G. Development of a critical care response - experiences from Italy during the Covid19 pandemic. Anesthesiol Clin. 2021;39(2):265-84.

4. Chong MSF, Hla TW, Sartori G. ICU surge capacity in a busy London district general hospital during the COVID-19 pandemic: Association of Anaesthetists; 2020. https://anaesthetists.org/Home/ Resources-publications/COVID-19-guidance/ICUsurge-capacity-in-a-busy-London-district-generalhospital-during-the-COVID-19-pandemic. Accessed 1 Apr 2021.

5. Turkiye Tourism Promotion and Development Agency. Covid-19 fight in Turkey 2020. https:// www.tga.gov.tr/turkiyenin-saglik-altyapisi-vecovid-19-yonetim-sureci/. Accessed 1 Apr 2021.

6. WHO. Attacks on health care in the context of COVID-19 2020 [updated 30 July 2020]. https:// www.who.int/news-room/feature-stories/detail/ attacks-on-health-care-in-the-context-of-covid-19. Accessed 3 Apr 2021.

7. COVID-19 update: FDA broadens emergency use authorization for veklury (remdesivir) to include all hospitalized patients for treatment of COVID-19 [press release]. FDA, August 28, 2020. https://www. fda.gov/news-events/press-announcements/covid19-update-fda-broadens-emergency-useauthorization-veklury-remdesivir-include-allhospitalized. Accessed 17 Dec 2020.

8. European Medicines Agency. Veklury (remdesivir). https://www.ema.europa.eu/en/medicines/human/ EPAR/veklury. Accessed 1 Apr 2021.

9. Lo MK, Jordan R, Arvey A, et al. GS-5734 and its parent nucleoside analog inhibit Filo-, Pneumo-, and paramyxoviruses. Sci Rep. 2017;7(1):43395.

10. Brown AJ, Won JJ, Graham RL, et al. Broad spectrum antiviral remdesivir inhibits human endemic and zoonotic deltacoronaviruses with a highly divergent RNA dependent RNA polymerase. Antiviral Res. 2019;169:104541.

11. Beigel JH, Tomashek KM, Dodd LE, et al. Remdesivir for the treatment of Covid-19 - final report. N Engl J Med. 2020;383(19):1813-26.

12. Hariyanto TI, Kwenandar F, Japar KV, Damay V, Kurniawan A. The effectiveness and safety of remdesivir for the treatment of patients with 
COVID-19: a systematic review and meta-analysis. Antiinfective Agents. 2021;19(3):333-40.

13. De Crescenzo F, Amato L, Cruciani F, et al. Comparative effectiveness of pharmacological interventions for Covid-19: a systematic review and network meta-analysis. Front Pharmacol. 2021;12:16.

14. Wang M, Wu T, Zuo Z, et al. Evaluation of current medical approaches for COVID-19: a systematic review and meta-analysis. BMJ Support Palliat Care. $2021 ; 11(1): 45-52$.

15. Wang Y, Zhang D, Du G, et al. Remdesivir in adults with severe COVID-19: a randomised, double-blind, placebo-controlled, multicentre trial. Lancet. 2020;395(10236):1569-78.

16. General Directorate of Public Health. Guideline for management of severe pneumonia, ARDS, sepsis and septic shock in COVID-19 October 23, 2020 ed. Ankara: Republic of Turkey Ministry of Health; 2020 .

17. General Directorate of Public Health. Guideline for management of COVID-19 adult patient. October 9, 2020 ed. Ankara, Turkey: Republic of Turkey Ministry of Health; 2020.

18. WHO Commission on Macroeconomics and Health. Macroeconomics and health: investing in health for economic development. Report of the Commission on Macroeconomics and Health. Geneva: World Health Organization; 2001.

19. Kohli M, Maschio M, Becker D, Weinstein MC. The potential public health and economic value of a hypothetical COVID-19 vaccine in the United States: use of cost-effectiveness modeling to inform vaccination prioritization. Vaccine. 2021;39(7): $1157-64$.
20. Hariyanto TI, Japar KV, Kwenandar F, et al. Inflammatory and hematologic markers as predictors of severe outcomes in COVID-19 infection: a systematic review and meta-analysis. Am J Emerg Med. 2021;41:110-9.

21. Gandhi RT, Lynch JB, Del Rio C. Mild or moderate Covid-19. N Engl J Med. 2020;383(18):1757-66.

22. General Directorate of Public Health. Management guideline for anticytokin-anti-inflammatory therapies and coagulopathies in COVID-19. November 7, 2020 ed. Ankara, Turkey: Republic of Turkey Ministry of Health; 2020.

23. Liu F, Li L, Xu M, et al. Prognostic value of interleukin-6, C-reactive protein, and procalcitonin in patients with COVID-19. J Clin Virol. 2020;127: 104370 .

24. Social Security Institution. Healthcare implementation notification (SUT 2013) 30 December 2020 ed. Legal Gazette of the Republic of Turkey: Presidency of the Republic of Turkey; 2021.

25. Jo Y, Jamieson L, Edoka I, et al. Cost-effectiveness of remdesivir and dexamethasone for COVID-19 treatment in South Africa. Open Forum Infect Dis. 2021. https://doi.org/10.1093/ofid/ofab040

26. Nichols BE, Jamieson L, Zhang SRC, et al. The role of remdesivir in South Africa: preventing COVID-19 deaths through increasing intensive care unit capacity. Clin Infect Dis. 2021; 72(9):1642-4.

27. Anderson MR, Bach PB, Baldwin MR. Hospital length of stay for patients with severe COVID-19: implications for remdesivir's value. PharmacoEconomics Open. 2021;5(1):129-31. 